Pacific Journal of Mathematics

sPATIALLY NORMAL RADICAL EXTENSIONS OF THE AVID ANDRew Gay, ANDRew MCDaniel and William Yslas Vélez 


\title{
PARTIALLY NORMAL RADICAL EXTENSIONS OF THE RATIONALS
}

\author{
David A. Gay, ANDREW MCDANIEL AND \\ WILLIAM YSLAS VÉLEZ
}

Dedicated to Henry B. Mann on the occasion of his retirement

If $K$ is a field and char $K \nmid n$, then any binomial $x^{n}-b \in K[x]$ has the property that $K(\alpha)$ is its splitting field for any root $\alpha$ iff a primitive $n$th root of unity $\zeta_{n}$ is an element of $K$. Thus, if $\zeta_{n} \in K$, any irreducible binomial $x^{n}-b \in K[x]$ is automatically normal. Similar nice results about binomials $x^{n}-b$ (Kummer theory comes to mind) can be obtained with the assumption $\zeta_{n} \in K$.

In this paper, without assuming the appropriate roots of unity are in $K$, one asks: what are the binomials $x^{m}-a \epsilon$ $K[x]$ having the property that $K(\alpha)$ is its splitting field for some root $\alpha$ ? Such binomials are called partially normal. General theorems are obtained in case $K$ is a real field. A complete list of partially normal binomials together with their Galois groups is found in case $K=Q$, the rational numbers.

This is a continuation of work begun in 1926 by Darbi [1] and Bessel-Hagen (see [8], p. 302) who determined all normal binomials over $\boldsymbol{Q}$. Recently, Mann and Vélez [5] considered binomials having a weaker property than normal but stronger than partially normal, namely, $K(\alpha)$ is the splitting field for any root $\alpha$. They obtained a complete classification of such binomials together with their Galois groups in case the ground field is $\boldsymbol{Q}$. In a similar direction, but for arbitrary ground field, Schinzel [7] has characterized two types of binomials: (a) those with abelian Galois group and (b) those whose polynomial degree is a power of a prime and which are products of normal factors.

The central role played by partially normal binomials in the general structure theory of pure extensions has been pointed out by Norris and Vélez in [6]. The results of Darbi and Bessel-Hagen, Mann and Vélez have been generalized for real fields by Gay [3].

In $\S 2$, we create a general setting by considering partially normal binomials over a typical real algebraic number field. (We have chosen this general setting to begin with in order to give more insight and to sketch how the results of this paper might be generalized to real fields.) In $\S 3$, we return to the rational numbers, apply the results of $\S 2$, and state a classification theorem. Sections 4 and 5 are devoted 
to proving this theorem. Finally, in $\S 6$, we compute the Galois groups of these binomials.

2. Generalities on partially normal binomial extensions of real fields. In what follows we let $R$ denote a fixed real algebraic number field. This section will be devoted to consequences of the following.

DEFINITION. The binomial $x^{m}-a \in R[x]$ is partially normal over $R$ if there is a root $\alpha$ of $x^{m}-a$ such that $R(\alpha)$ is the splitting field of $x^{m}-a$ over $R$. Such a root $\alpha$ is called a generating root.

Let $\zeta_{n}$ be a primitive $n$th root of unity. Let $\phi_{R}(n)=\left[R\left(\zeta_{n}\right): R\right]$. Thus $\phi_{R}$ is an Euler's $\phi$-function relative to the field $R$. In particular, $\phi_{Q}(n)=\phi(n)$.

Proposition 2.1. The binomial $x^{m}-a \in R[x]$ is partially normal over $R$ iff there exists a root $\gamma$ of $x^{m}-a$ such that $R\left(\zeta_{m}\right) \subseteq R(\gamma)$. Furthermore, if $x^{m}-a$ is partially normal over $R$ with generating root $\alpha$, then there is a positive integer s such that $[R(\alpha): R]=s \phi_{R}(m)$, $R\left(\alpha^{s}\right)=R\left(\zeta_{m}\right)$ and $x^{s}-\alpha^{s}$ is the minimal polynomial for $\alpha$ over $R\left(\zeta_{m}\right)$.

Proof. The first statement of the proposition follows from the fact that if $\delta$ is a root of $x^{m}-a$ then all the roots are $\delta, \zeta_{m} \delta, \zeta_{m}^{2} \delta$, $\cdots, \zeta_{m}^{m-1} \delta$.

To prove the second statement, we note that from the first statement $R\left(\zeta_{m}\right) \subseteq R(\alpha)$. Let $s=\left[R(\alpha): R\left(\zeta_{m}\right)\right]$. Then $[R(\alpha): R]=$ $s \phi_{R}(m)$. Now let $f(x)$ be the minimal polynomial for $\alpha$ over $R\left(\zeta_{m}\right)$. Then $f(x)$ is of degree $s$ and

$$
f(x)=\prod_{j=1}^{s}\left(x-\zeta_{m}^{i_{j}} \alpha\right) .
$$

The constant term of $f(x)= \pm \alpha^{s} \amalg_{j=1}^{s} \zeta_{m}^{i_{j}}$ and is in $R\left(\zeta_{m}\right)$. Thus $\alpha^{s} \in$ $R\left(\zeta_{m}\right)$. Since $x^{s}-\alpha^{s}$ is a polynomial of degree $s$ over $R\left(\zeta_{m}\right)$ with $\alpha$ as a root, $f(x)=x^{s}-\alpha^{s}$. This together with the fact $R\left(\alpha^{s}\right) \leqq R\left(\zeta_{m}\right)$ forces $R\left(\alpha^{s}\right)=R\left(\zeta_{m}\right)$.

In what follows, we choose a fixed binomial $x^{m}-a$, partially normal over $R$ with generating root $\alpha$ and $s=\left[R(\alpha): R\left(\zeta_{m}\right)\right]$ as in Proposition 2.1. Let $\beta=\sqrt[m]{|a|}$, the real, positive $m$ th root of $|a|$. Let $q$ be the smallest positive integer such that $\beta^{q} \in R$. The following results will tell us something about the relationships among $\beta, s$ and $q$ and their limitations. First, two lemmas. 
LEMma 2.2. Let $\gamma$ be algebraic over a field $F$ with $\gamma^{r} \in F$ and $x^{r}-\gamma^{r}$ irreducible over $F$. Then $\gamma^{t} \in F$ ( $t$ a positive integer) implies $r \mid t$.

Proof. If $\gamma^{r} \in F$ and $x^{r}-\gamma^{r}$ irreducible over $F$, then $[F(\gamma): F]=$ $r$. Thus, if $\gamma^{t} \in F, t \geqq r$. If $r \nmid t$, then write $t=a r+b$ with $0<$ $b<r$. Hence $\gamma^{t}=\left(\gamma^{r}\right)^{a} \gamma^{b}$ or $\gamma^{b} \in F$, a contradiction. Thus $r \mid t$.

Lemma 2.3. Let $K$ be an abelian extension of the real field $R$. Suppose $a \in R$ with $a>0$ and $\sqrt[m]{a} \in K(\sqrt[m]{a}$ is a real $m$ th root). Then $\left(\sqrt[m]{a)^{2}} \in R\right.$

Proof. This is a standard result in Galois theory. See [5], Lemma 1.

Proposition 2.4. We have (a) $s \mid m$ and $q \mid m$; (b) $\beta^{2 s} \in R$; (c) if $\beta^{s} \in R$, then $q \mid s$ and if $\beta^{s} \notin R$, then $q \mid 2 s$.

Proof. (a) From Proposition $2.1 x^{s}-\alpha^{s}$ is irreducible over $R\left(\zeta_{m}\right)$. But also $\alpha^{m}=a \in R$. Thus from Lemma 2.2, $s \mid m$. By an argument similar to the proof of Lemma 2.2, $q$ divides any positive integer $t$ such that $\beta^{t} \in R$. Thus $q \mid m$.

(b) Now $\alpha=\zeta_{m}^{j} \zeta_{2 m}^{\varepsilon} \beta$ where $\varepsilon=0$ if $a>0$ and $\varepsilon=1$ if $a<0$. Thus $\alpha^{s}=\zeta_{m}^{s j \zeta_{2 m}^{s \varepsilon}} \beta^{s} \in R\left(\zeta_{m}\right)$ by Proposition 2.1. Hence $\beta^{s} \in R\left(\zeta_{2 m}\right)$. By Lemma 2.3, $\beta^{s}$ is either an element of $R$ or is the square root of an element of $R$. In either case, $\beta^{2 s} \in R$.

(c) By (b) and the proof of part (a), $q \mid 2 s$. If, in addition, $\beta^{s} \in R$, then $q \mid s$.

The following result delineates $q$ and $s$ more precisely:

Proposition 2.5. (a) If $a>0$ or $a<0$ and $q$ even, then $\beta^{s} \in R$ iff $q=s$.

(b) If $a<0$ and $q$ odd, then $\beta^{s} \in R$ iff $q=s$ or $s=2 q$.

(c) $\beta^{s} \notin R$ iff $q=2 s$.

Proof. (a) Suppose $\beta^{s} \in R$ and $a>0$ or $a<0$ and $q$ even. Then $\alpha^{q}=\zeta_{m}^{q J} \zeta_{2 m}^{\varepsilon q} \beta^{q} \in R\left(\zeta_{m}\right)$. Hence, by Proposition 2.1 and Lemma 2.2, $s \mid q$. By Proposition 2.4 (c), $q \mid s$. Thus $q=s$. The converse is obvious.

(b) If $\beta^{s} \in R, a<0$ and $q$ odd, then $\alpha^{2 q}=\zeta_{m}^{2 q j} \zeta_{m}^{q} \beta^{2 q} \in R\left(\zeta_{m}\right)$. Thus by Proposition 2.1 and Lemma 2.2, $s \mid 2 q$. But by Proposition 2.4 (c), $q \mid s$. Thus $s=q$ or $s=2 q$. Again the converse is obvious.

(c) Suppose $\beta^{s} \notin R$. Thus by Proposition 2.4 (c), $q \mid 2 s$. Furthermore, $q$ must be even. Thus $\alpha^{q} \in R\left(\zeta_{m}\right)$. Again by Lemma 2.2, $s \mid q$. 
Thus $s=q$ or $2 s=q$. The former cannot happen since $\beta^{s} \notin R$. Thus $2 s=q$. The converse is easy.

The following result will enable us to narrow the possibilities for $s$ considerably.

Proposition 2.6. In all cases $\dot{\phi}_{R}(m) \leqq 2 \phi_{R}(m / s)$. In particular, if $\alpha>0$ and $\beta^{s} \in R$, then $\phi_{R}(m)=\phi_{R}(m / s)$; if $\alpha<0$, and $\beta^{s} \in R$, then $\phi_{R}(m)=\phi_{R}(2 m / s)$.

Proof. We have $\alpha=\zeta_{m}^{j} \zeta_{2 m}^{\varepsilon} \beta$. Thus $\alpha^{2 s}=\zeta_{m / s}^{2 j} \zeta_{m / s}^{\varepsilon} \beta^{2 s} \in R\left(\zeta_{m / s}\right)$. Consequently, $s \phi_{R}(m)=[R(\alpha): R]=\left[R(\alpha): R\left(\zeta_{m / s}\right)\right]\left[R\left(\zeta_{m / s}\right): R\right] \leqq 2 s \dot{\phi}_{R}(m / s)$ or $\phi_{R}(m) \leqq 2 \phi_{R}(m / s)$.

If $a>0$ and $\beta^{s} \in R$, then $\alpha^{s}=\zeta_{m / s}^{j} \beta^{s} \in R\left(\zeta_{m / s}\right)$. Thus $\dot{\phi}_{R}(m)=$ $\phi_{R}(m / s)$. If $s=2 q$, then by $2.5 \beta^{s} \in R$ and $\alpha^{s}=\zeta_{m / s}^{j} \zeta_{m / q} \beta^{s} \in R\left(\zeta_{m / q}\right)$. Thus $\phi_{R}\left(m\left(=\phi_{R}(m / q)=\phi_{R}(2 m / s)\right.\right.$. Finally, if $\alpha<0, \beta^{s} \in R$ and $q=s$, then $\alpha^{s}=\zeta_{m / s}^{j} \zeta_{2 m / s} \beta^{s} \in R\left(\zeta_{2 m / s}\right) \subseteq R\left(\zeta_{m}\right)$. Thus $\phi_{R}(m)=\phi_{R}(2 m / s)$.

3. Partially normal pure extensions of the rationals. In this section we will apply the results of $\S 2$ to the field $R=\boldsymbol{Q}$. Without loss of generality, we consider (as in [4]) only those binomials $x^{n}-a$ with $a$ an integer. The following lemma will enable us to use Proposition 2.6 directly. We use the notation $p^{a} \| m$, for prime $p$, to mean $p^{\alpha} \mid m$ but $p^{\alpha+1} \nmid m$.

LEMMA 3.1. Let $d$ and $m$ be positive integers with $d \mid m$.

(a) If $\phi(m) \leqq 2 \phi(m / d)$, then $d=1,2,3,4$ or 6 . In case $d=6$, then $2 \| m$; if $3 \mid d$, then $3 \| m$; if $d=4$, then $4 \| m$.

(b) If $\phi(m)=\phi(m / d)$, then $d=1$ or 2 . In case $d=2$, then $2 \| m$.

Proof. Part (b) is obvious. To prove part (a), we first make an observation. Suppose one of the following occurs:

$$
\begin{aligned}
\text { either } & p \mid d \text { with } p \text { a prime }>3, \\
\text { or } & 9 \mid m \text { and } 3 \mid d, \\
\text { or } & 8 \mid d, \\
\text { or } & 4|| d \text { and } 8 \mid m, \\
\text { or } & 6 \mid d \text { and } 4 \mid m .
\end{aligned}
$$

Then $\phi(m) \geqq 3 \dot{\phi}(m / d)$. Thus, in order that $\dot{\phi}(m) \leqq 2 \phi(m / d)$ be true we must have

(i ) $d=1,2,3,4,6$ or 12 ; (ii) if $3 \mid d$ then $3 \| m$; (iii) if $d=4$, then $4 \| m$; and (iv) if $d=6$, then $2 \| m$. The case $d=12$ is impossible, as is easily checked. 
COROLlaRY 3.2. If $x^{m}-\alpha$ is partially normal over $\boldsymbol{Q}$, then $s=1,2,3,4$, or 6 .

The following result limits $s$ even more and relates its value to the rationality of $\beta^{s}$.

Proposition 3.3. (a) $\beta^{s} \in \boldsymbol{Q}$ and $a>0$ implies $s=1,2(s=q)$.

(b) $\beta^{s} \in \boldsymbol{Q}$ and $a<0$ implies $s=1,2(s=q)$ or $s=2, q=1$.

(c) $\beta^{s} \notin \boldsymbol{Q}$ implies $s=1,2$ or $3(q=2 s)$.

[We shall see later that all these possibilities can actually be realized.]

Proof. (a) By Proposition 2.6 and Lemma 3.1, $s=1$ or 2 . By $2.5, q=s$.

(b) If $a<0$ and $\beta^{s} \in R$, then by $2.6 \phi(m)=\phi(2 m / s)$. If $q$ is even, then $q=s=2 s_{1}$, by 2.5. Thus by 3.1 (b) $s_{1}=1$ or 2 . If $s_{1}=$ 2 , then $2 \| m$. But $s=4$ implies $4 \mid m$ (2.4). This is a contradiction. Thus $q=s=2$. If $q$ is odd, then by 2.5 (b) $q=s$ or $2 q=s$. If $q=s$, then $\boldsymbol{Q}\left(\zeta_{m}\right)=\boldsymbol{Q}\left(\alpha^{q}\right)=\boldsymbol{Q}\left(\zeta_{m / q}^{j} \zeta_{2 m / q}\right) \subseteq \boldsymbol{Q}\left(\zeta_{2 m / q}\right)$. This can happen for $q$ odd only when $q=1$. On the other hand, if $s=2 q$, then $\phi(m)=$ $\phi(m / q)$ so that, by $3.1(\mathrm{~b}), q=1(s=2)$ or $q=2(s=4)$. If $q=2$, then $2 \| m$. But again $s=4$ implies $4 \mid m$, a contradiction. Thus $q=1, s=2$. This completes the proof of (b).

(c) If $\beta^{s} \notin \boldsymbol{Q}$, then we know $\phi(m) \leqq 2 \phi(m / s)(2.6)$ and $q=2 s(2.5$ (c)). From the first statement we know $s=1,2,3,4$ or 6 (3.1). But if $s=4$, then $q=8$. Since $q \mid \mathrm{m}$, this contradicts 3.1. Similarly, if $s=6$, then $q=12$ and $12 \mid \mathrm{m}$ contradicting 3.1. Thus $s=4$ and $s=6$ are impossible. We conclude that $s=1,2,3$ with $q=2 s$.

COROLlaRY 3.4. If $x^{m}-a$ is partially normal over $\boldsymbol{Q}$, then there is a positive integer $b$ such that either $a= \pm b^{m}, a= \pm b^{m / 2}$, $a= \pm b^{m / 4}$, or $a= \pm b^{m / 6}$.

Corollary 3.4 marks out the possibilities for partially normal binomials over $\boldsymbol{Q}$ quite clearly. The following theorem (our main result) gives necessary and sufficient conditions for when one of these admissible binomials is actually partially normal.

THEOREM 3.5. Let $m$ and $b$ be positive integers. Then

(1) $x^{m}-b^{m}$ is partially normal with $s=q=1 ; x^{m}+b^{m}$ is partially normal with $s=2, q=1$ ( $m$ even) or $s=q=1$ ( $m$ odd).

(2) $x^{m}-b^{m / 2}$ is partially normal with $s=q=2 \Leftrightarrow 2 \| m$ and $\sqrt{b} \notin \boldsymbol{Q}\left(\zeta_{m}\right)$. 
(3) $x^{m}-b^{m / 2}$ is partially normal with $s=1, q=2 \Leftrightarrow$ either $\sqrt{b} \in \boldsymbol{Q}\left(\zeta_{m / 2}\right)$ and $\sqrt{b \notin \boldsymbol{Q}}$ or $\sqrt{b} \in \boldsymbol{Q}\left(\zeta_{m}\right), \sqrt{b} \notin \boldsymbol{Q}\left(\zeta_{m / 2}\right)$ and $4 \| m$.

(4) $x^{m}+b^{m / 2}$ is partially normal with $s=1, q=2 \Leftrightarrow \sqrt{b} \in Q\left(\zeta_{2 m}\right)$ and $\sqrt{b} \notin \boldsymbol{Q}\left(\zeta_{m}\right)$.

(5) $x^{m}+b^{m / 2}$ is partially normal with $s=q=2 \Leftrightarrow \sqrt{b} \notin \boldsymbol{Q}\left(\zeta_{2 m}\right)$ or $\sqrt{b} \in \boldsymbol{Q}\left(\zeta_{m}\right)$ and $\sqrt{b} \notin \boldsymbol{Q}$.

(6) $x^{m}-b^{m / 4}$ is partially normal with $s=2, q=4 \Leftrightarrow \sqrt{b} \epsilon$ $\boldsymbol{Q}\left(\zeta_{m}\right), \sqrt{b} \notin \boldsymbol{Q}\left(\zeta_{m / 2}\right)$ and $4 \| m$.

(7) $x^{m}+b^{m / 4}$ is partially normal with $s=2, q=4 \Leftrightarrow \sqrt{b} \epsilon$ $\boldsymbol{Q}\left(\zeta_{m / 2}\right)$ and $\sqrt{b} \notin \boldsymbol{Q}$.

(8) $x^{m}-b^{m / 6}$ is partially normal with $s=3, q=6 \Leftrightarrow \sqrt{b} \epsilon$ $\boldsymbol{Q}\left(\zeta_{m}\right), \sqrt{b} \notin \boldsymbol{Q}\left(\zeta_{m / 3}\right)$ and $2 \| m$.

(9) $x^{m}+b^{m / 6}$ is partially normal with $s=3, q=6 \Leftrightarrow \sqrt{b} \epsilon$ $\boldsymbol{Q}\left(\zeta_{2 m}\right), \sqrt{b} \notin \boldsymbol{Q}\left(\zeta_{m}\right)$ and $\sqrt{b} \notin \boldsymbol{Q}\left(\zeta_{2 m / 3}\right)$.

We will prove this theorem in $\S 5$ after stating and proving some useful lemmas.

4. Square roots and generators of cyclotomic extensions. In this section we will state and prove some results which will be used to prove our main Theorem 3.5. These results are independent of the rest of this paper and are partial responses to the following question: for what positive integers $b, m, n$ is it the case that $\boldsymbol{Q}\left(\zeta_{m} \sqrt{b}\right)=\boldsymbol{Q}\left(\zeta_{n}\right)$ ? We will use (implicitly) the following known result from Galois theory. (See, for example, [2] p. 240.)

LEMMA 4.1.

(A) If $p$ is an odd prime, then $\sqrt{p} \in \boldsymbol{Q}\left(\zeta_{p}\right)$ iff $p \equiv 1(\bmod 4)$ and $\sqrt{-p} \in \boldsymbol{Q}\left(\zeta_{p}\right)$ iff $p \equiv 3(\bmod 4) ; \sqrt{-1} \in \boldsymbol{Q}\left(\zeta_{4}\right) ; \sqrt{\mathbf{2}} \in \boldsymbol{Q}\left(\zeta_{8}\right)$.

(B) Let $b$ be a square free integer. Then, if $m$ is odd, $b \mid m$ iff $\sqrt{b}$ or $\sqrt{-b} \in \boldsymbol{Q}\left(\zeta_{m}\right)=\boldsymbol{Q}\left(\zeta_{2 m}\right)$ iff $\sqrt{b} \in \boldsymbol{Q}\left(\zeta_{4 m}\right)$ iff $\sqrt{-b} \in \boldsymbol{Q}\left(\zeta_{4 m}\right)$. Further, if $8 \mid m$, then $\sqrt{b} \in \boldsymbol{Q}\left(\zeta_{m}\right)$ iff $b \mid m$.

LEMMA 4.2. Let $m$ and $b$ be positive integers. Then

(A) If $m$ is even, then $\sqrt{b} \in \boldsymbol{Q}\left(\zeta_{2 m}\right), \sqrt{b} \notin \boldsymbol{Q}\left(\zeta_{m}\right)$, and $\sqrt{b} \notin \boldsymbol{Q}\left(\zeta_{2 m / 3}\right)$ iff $\boldsymbol{Q}\left(\zeta_{2 m / 3} \sqrt{b}\right)=\boldsymbol{Q}\left(\zeta_{m}\right)$.

(B) If $m$ is odd, then $\sqrt{b} \in \boldsymbol{Q}\left(\zeta_{m}\right), \sqrt{b} \notin \boldsymbol{Q}\left(\zeta_{m / 3}\right)$ iff $\boldsymbol{Q}\left(\zeta_{m / 3} \sqrt{b}\right)=$ $\boldsymbol{Q}\left(\zeta_{m}\right)$

Proof. (A) It is sufficient to consider the case where $b$ is square free. Now $\sqrt{b} \in \boldsymbol{Q}\left(\zeta_{2 m}\right)$ and $\sqrt{b} \notin \boldsymbol{Q}\left(\zeta_{2 m / 3}\right)$ implies $3 \| m$. Similarly, $\sqrt{b} \in \boldsymbol{Q}\left(\zeta_{2 m}\right)$ and $\sqrt{b} \notin \boldsymbol{Q}\left(\zeta_{m}\right)$ implies $8 \nmid m$. Thus there are two possibilities: (a) $2 \| m$ in which case $b=3 b_{1}$ with $\left(b_{1}, 6\right)=1$ ! and $\sqrt{b_{1}} \epsilon$ $\boldsymbol{Q}\left(\zeta_{m / 3}\right)$ and (b) $4 \| m$ in which case $b=6 b_{1}$ with $\left(b_{1}, 6\right)=1$ and $\sqrt{b_{1}} \in$ 
$\boldsymbol{Q}\left(\zeta_{m / 3}\right)$. In both cases $\boldsymbol{Q}\left(\zeta_{2 m / 3} \sqrt{b}\right)$ is an extension of $\boldsymbol{Q}\left(\zeta_{m / 3}\right)$. In case (a),

$$
\begin{aligned}
\boldsymbol{Q}\left(\zeta_{2 m / 3} \sqrt{\bar{b})}\right. & =\boldsymbol{Q}\left(\zeta_{m / 3}, \zeta_{4} \zeta_{m / 6} \sqrt{3} \sqrt{b_{1}}\right) \\
& =\boldsymbol{Q}\left(\zeta_{m / 3}, \zeta_{4} \sqrt{3}\right) \\
& =\boldsymbol{Q}\left(\zeta_{m / 3}, \sqrt{-3}\right) \\
& =\boldsymbol{Q}\left(\zeta_{m}\right)
\end{aligned}
$$

and, in case (b),

$$
\begin{aligned}
\boldsymbol{Q}\left(\zeta_{2 m / 3} \sqrt{\bar{b})}\right. & =\boldsymbol{Q}\left(\zeta_{m / 3}, \zeta_{8} \zeta_{m / 12} \sqrt{2} \sqrt{3} \sqrt{b_{1}}\right) \\
& =\boldsymbol{Q}\left(\zeta_{m / 3}, \zeta_{8} \sqrt{2} \sqrt{3}\right) \\
& =\boldsymbol{Q}\left(\zeta_{m / 3},(1+i) \sqrt{3}\right) \\
& =\boldsymbol{Q}\left(\zeta_{m / 3}, \sqrt{3}\right) \\
& =\boldsymbol{Q}\left(\zeta_{m}\right) .
\end{aligned}
$$

Conversely, suppose $\boldsymbol{Q}\left(\zeta_{2 m / 3} \sqrt{\bar{b})}=\boldsymbol{Q}\left(\zeta_{m}\right)\right.$. Thus $\zeta_{2 m / 3} \sqrt{b} \in \boldsymbol{Q}\left(\zeta_{2 m}\right)$ and, therefore, $\sqrt{b} \in \boldsymbol{Q}\left(\zeta_{2 m}\right)$. If also $\sqrt{b} \in \boldsymbol{Q}\left(\zeta_{m}\right)$, then also $\zeta_{2 m / 3} \in \boldsymbol{Q}\left(\zeta_{m}\right)$ contradicting the fact that $m$ is even. Thus $\sqrt{b} \notin \boldsymbol{Q}\left(\zeta_{m}\right)$. Finally, if $\sqrt{b} \in \boldsymbol{Q}\left(\zeta_{2 m / 3}\right)$, then $\boldsymbol{Q}\left(\zeta_{m}\right) \subseteq \boldsymbol{Q}\left(\zeta_{2 m / 3}\right)$ which is impossible. Thus also $\sqrt{b} \notin \boldsymbol{Q}\left(\zeta_{2 m / 3}\right)$. This completes the proof of $(\mathrm{A})$.

(B) Again we may assume without loss of generality that $b$ is square free. Now $\sqrt{b} \in \boldsymbol{Q}\left(\zeta_{2 m}\right)$ and $\sqrt{b} \notin \boldsymbol{Q}\left(\zeta_{2 m / 3}\right)$ implies $3 \| m, b=3 b_{1}$ and $\sqrt{-b_{1}} \in \boldsymbol{Q}\left(\zeta_{2 m / 3}\right)=\boldsymbol{Q}\left(\zeta_{m / 3}\right)$. Thus, since $\boldsymbol{Q}\left(\zeta_{2 m / 3} \sqrt{\bar{b})}\right.$ is an extension of $\boldsymbol{Q}\left(\zeta_{m / 3}\right)$, we have

$$
\begin{aligned}
\boldsymbol{Q}\left(\zeta_{2 m / 3} \sqrt{b)}\right. & =\boldsymbol{Q}\left(\zeta_{m / 3}, \sqrt{-3} \sqrt{\left.-b_{1}\right)}\right. \\
& =\boldsymbol{Q}\left(\zeta_{m / 3}, \sqrt{-3}\right) \\
& =\boldsymbol{Q}\left(\zeta_{m}\right) .
\end{aligned}
$$

The proof of the converse is analogous to that of (A).

Lemma 4.3. Let $m$ and $b$ be positive integers with $m$ even. Then

(A) The following statements are equivalent

(i) $\sqrt{b} \in \boldsymbol{Q}\left(\zeta_{2 m}\right)$ and $\sqrt{b} \notin \boldsymbol{Q}\left(\zeta_{m}\right)$

(ii) $\boldsymbol{Q}\left(\zeta_{2 m} \sqrt{\bar{b})}=\boldsymbol{Q}\left(\zeta_{m}\right)\right.$

(iii) either $2 \| m$, the square free part of $b$ is odd and $\sqrt{-b} \epsilon$ $\boldsymbol{Q}\left(\zeta_{m}\right)$ or $4 \| m$, the square free part of $b$ is even and $\sqrt{b / 2} \in \boldsymbol{Q}\left(\zeta_{m}\right)$.

(B) $2 \| m, \sqrt{b} \in \boldsymbol{Q}\left(\zeta_{2 m}\right)$ and $\sqrt{b} \notin \boldsymbol{Q}\left(\zeta_{m}\right) \Leftrightarrow \boldsymbol{Q}\left(\zeta_{m} \sqrt{b)}=\boldsymbol{Q}\left(\zeta_{2 m}\right)\right.$.

Proof. (A) We will show that (i) $\Rightarrow$ (iii) $\Rightarrow$ (ii) $\Rightarrow$ (i). Suppose $\sqrt{b} \in \boldsymbol{Q}\left(\zeta_{2 m}\right)$ and $\sqrt{b} \notin \boldsymbol{Q}\left(\zeta_{m}\right)$. Thus $8 \nmid m$. It follows that, since $m$ is even, either $2 \| m$ or $4 \| m$. In the first case it also follows that 
the square free part of $b$ must be odd and that $\sqrt{-b} \in \boldsymbol{Q}\left(\zeta_{m}\right)$. In the second case it also follows that the square free part of $b$ is even and $\sqrt{b / 2} \in \boldsymbol{Q}\left(\zeta_{m}\right)$. This shows that (i) implies (iii).

Now assume that (iii) holds. Clearly $\boldsymbol{Q}\left(\zeta_{2 m} \sqrt{b}\right)$ is an extension of $\boldsymbol{Q}\left(\zeta_{m}\right)$. Thus, in case $2 \| m$,

$$
\begin{aligned}
\boldsymbol{Q}\left(\zeta_{2 m} \sqrt{b)}\right. & =\boldsymbol{Q}\left(\zeta_{m}, \zeta_{2 m} \sqrt{b}\right) \\
& =\boldsymbol{Q}\left(\zeta_{m}, \zeta_{4} \zeta_{m / 2} \sqrt{b)}\right. \\
& =\boldsymbol{Q}\left(\zeta_{m}, \sqrt{-b}\right) \\
& =\boldsymbol{Q}\left(\zeta_{m}\right) .
\end{aligned}
$$

In case $4 \| m$,

$$
\begin{aligned}
\boldsymbol{Q}\left(\zeta_{2 m} \sqrt{\bar{b}}\right) & =\boldsymbol{Q}\left(\zeta_{m}, \zeta_{8} \zeta_{m / 4} \sqrt{2} \sqrt{\frac{b}{2}}\right) \\
& =\boldsymbol{Q}\left(\zeta_{m}, \zeta_{8} \sqrt{2}\right) \\
& =\boldsymbol{Q}\left(\zeta_{m}\right) .
\end{aligned}
$$

This shows that (iii) implies (ii).

That (ii) implies (i) is obvious. This completes the proof of part (A) of the lemma.

(B) If $2 \| m, \sqrt{b} \in \boldsymbol{Q}\left(\zeta_{2 m}\right)$ and $\sqrt{b} \notin \boldsymbol{Q}\left(\zeta_{m}\right)$, then $\sqrt{-b} \in \boldsymbol{Q}\left(\zeta_{m}\right)$. Furthermore, $\boldsymbol{Q}\left(\zeta_{m} \sqrt{b}\right)$ is an extension of $\boldsymbol{Q}\left(\zeta_{m / 2}\right)=\boldsymbol{Q}\left(\zeta_{m}\right)$. Thus

$$
\begin{aligned}
\boldsymbol{Q}\left(\zeta_{m} \sqrt{b)}\right. & =\boldsymbol{Q}\left(\zeta_{m / 2}, \zeta_{m} \sqrt{b}\right) \\
& =\boldsymbol{Q}\left(\zeta_{m / 2}, \sqrt{b)}\right. \\
& =\boldsymbol{Q}\left(\zeta_{m / 2}, i \sqrt{-b}\right) \\
& =\boldsymbol{Q}\left(\zeta_{m / 2}, i\right) \\
& =\boldsymbol{Q}\left(\zeta_{2 m}\right) .
\end{aligned}
$$

Conversely, suppose $\boldsymbol{Q}\left(\zeta_{m} \sqrt{b)}=\boldsymbol{Q}\left(\zeta_{2 m}\right)\right.$. It follows easily that $\sqrt{b} \in \boldsymbol{Q}\left(\zeta_{2 m}\right)$. If also $\sqrt{b} \in \boldsymbol{Q}\left(\zeta_{m}\right)$, then $\boldsymbol{Q}\left(\zeta_{2 m}\right) \subseteq \boldsymbol{Q}\left(\zeta_{m}\right)$ contradicting the fact that $m$ is even. Thus $\sqrt{b} \in \boldsymbol{Q}\left(\zeta_{2 m}\right)$ and $\sqrt{b} \notin \boldsymbol{Q}\left(\zeta_{m}\right)$. By part (A) of the lemma, either $2 \| m$ or $4 \| m$. We will show that the latter is impossible. Indeed, suppose $4 \| m$ and $\boldsymbol{Q}\left(\zeta_{m} \sqrt{b)}=\boldsymbol{Q}\left(\zeta_{2 m}\right)\right.$. On the one hand, $\boldsymbol{Q}\left(\zeta_{2 m}\right)$ is an extension of $\boldsymbol{Q}\left(\zeta_{m / 2}\right)$ of degree 4 . On the other hand, $\boldsymbol{Q}\left(\zeta_{m} \sqrt{b)}\right.$ is an extension of $\boldsymbol{Q}\left(\zeta_{m / 2}\right)$ of degree at most 2. Thus $4 \| m$ cannot happen and part (B) of the lemma follows.

\section{Proof of Theorem 3.5.}

(1) This is easy.

(2) In this case $\alpha=\zeta_{m}^{j} \sqrt{b}$ and thus $\boldsymbol{Q}\left(\alpha^{2}\right)=\boldsymbol{Q}\left(\zeta_{m}^{2 j}\right)=\boldsymbol{Q}\left(\zeta_{m}\right)$. Consequently, $2 \| m$ and $\sqrt{b} \notin \boldsymbol{Q}\left(\zeta_{m}\right)$. 
Conversely, if $2 \| m$ and $\sqrt{b} \notin \boldsymbol{Q}\left(\zeta_{m}\right)$, then $\boldsymbol{Q}\left(\zeta_{m} \sqrt{b}\right)$ is an extension of $\boldsymbol{Q}\left(\zeta_{m}\right)$ of degree exactly 2 .

(3) In this case, $\alpha=\zeta_{m}^{j} \sqrt{b}, s=1$ and $\boldsymbol{Q}(\alpha)=\boldsymbol{Q}\left(\zeta_{m}\right)$. Thus $\sqrt{b} \in \boldsymbol{Q}\left(\zeta_{m}\right)$. If also $\sqrt{b} \notin \boldsymbol{Q}\left(\zeta_{m / 2}\right)$, then by Lemma 4.3 (A), $\boldsymbol{Q}\left(\zeta_{m} \sqrt{b)}=\right.$ $\boldsymbol{Q}\left(\zeta_{m / 2}\right)$. Thus $(j, m)=d \neq 1$. Consequently, $\boldsymbol{Q}\left(\zeta_{m}\right)=\boldsymbol{Q}\left(\zeta_{m / d} \sqrt{b)}=\right.$ $\boldsymbol{Q}\left(\zeta_{m / d}, \sqrt{b}\right)$ is an extension of degree 2 over $\boldsymbol{Q}\left(\zeta_{m / d}\right)$. This can happen only when $d=2$ or 3 . If $d=3$, then by Lemma 4.2 $\boldsymbol{Q}\left(\zeta_{m / 3} \sqrt{b}\right)=\boldsymbol{Q}\left(\zeta_{m / 2}\right)$ which is a contradiction. On the other hand, if $d=2$, then $\boldsymbol{Q}\left(\zeta_{m / 2} \sqrt{b}\right)=\boldsymbol{Q}\left(\zeta_{m}\right)$. This and Lemma 4.3 (B) then implies $4 \| m$.

Conversely, suppose $\sqrt{b} \in \boldsymbol{Q}\left(\zeta_{m / 2}\right)$. Then $\boldsymbol{Q}\left(\zeta_{m} \sqrt{b}\right)$ is an extension of $\boldsymbol{Q}\left(\zeta_{m / 2}\right)$ and $\boldsymbol{Q}\left(\zeta_{m} \sqrt{b}\right)=\boldsymbol{Q}\left(\zeta_{m / 2}, \zeta_{m} \sqrt{b}\right)=\boldsymbol{Q}\left(\zeta_{m / 2}, \zeta_{m}\right)=\boldsymbol{Q}\left(\zeta_{m}\right)$. Thus $\alpha=\zeta_{m} \sqrt{b}$ generates the splitting field of $x^{m}-b^{m / 2}$ which is therefore p.n. (partially normal) with $s=1$. On the other hand, suppose $\sqrt{b} \epsilon$ $\boldsymbol{Q}\left(\zeta_{m}\right), \sqrt{b} \notin \boldsymbol{Q}\left(\zeta_{m / 2}\right)$ and $4 \| m$. Then by Lemma $4.3(\mathrm{~B}), \boldsymbol{Q}\left(\zeta_{m / 2} \sqrt{b}\right)=$ $\boldsymbol{Q}\left(\zeta_{m}\right)$ so that $x^{m}-b^{m / 2}$ is p.n. with $s=1$.

(4) If $\alpha=\zeta_{m}^{j} \zeta_{2 m} \sqrt{b}$ generates the splitting field of $x^{m}+b^{m / 2}$ with $\boldsymbol{Q}(\alpha)=\boldsymbol{Q}\left(\zeta_{m}\right)$, then clearly $\sqrt{b} \in \boldsymbol{Q}\left(\zeta_{2 m}\right)$ and $\sqrt{b} \notin \boldsymbol{Q}\left(\zeta_{m}\right)$.

Conversely, suppose $\sqrt{b} \in \boldsymbol{Q}\left(\zeta_{2 m}\right)$ and $\sqrt{b} \notin \boldsymbol{Q}\left(\zeta_{m}\right)$. Then by Lemma $4.3(\mathrm{~A}), \boldsymbol{Q}\left(\zeta_{2 m} \sqrt{b)}=\boldsymbol{Q}\left(\zeta_{m}\right)\right.$. Hence $x^{m}+b^{m / 2}$ is p.n. with $s=1$.

(5) Suppose that $\alpha=\zeta_{m}^{j} \zeta_{2 m} \sqrt{b}$ generates the splitting field of $x^{m}+b^{m / 2}$ and $\boldsymbol{Q}\left(\alpha^{2}\right)=\boldsymbol{Q}\left(\zeta_{m}^{2 j} \zeta_{m}\right)=\boldsymbol{Q}\left(\zeta_{m}\right)$. Then either $\bar{V} b \in \boldsymbol{Q}\left(\zeta_{m}\right)$ or $\sqrt{b} \notin \boldsymbol{Q}\left(\zeta_{m}\right)$. If the latter, then $\sqrt{b} \notin \boldsymbol{Q}\left(\zeta_{2 m}\right)$ also. For, if $\sqrt{b} \in \boldsymbol{Q}\left(\zeta_{2 m}\right)$ and $\notin \boldsymbol{Q}\left(\zeta_{m}\right)$, then by Lemma 4.3 (A) $\boldsymbol{Q}\left(\zeta_{2 m} \sqrt{b}\right)=\boldsymbol{Q}\left(\zeta_{m}\right)$. It would then follow that $\boldsymbol{Q}(\alpha)=\boldsymbol{Q}\left(\zeta_{m}\right)$ contradicting the fact that $s=2$. Thus either $\sqrt{b} \in \boldsymbol{Q}\left(\zeta_{m}\right)$ or $\sqrt{b} \notin \boldsymbol{Q}\left(\zeta_{2 m}\right)$.

Conversely, suppose $\sqrt{b} \in \boldsymbol{Q}\left(\zeta_{m}\right)$. Then $\boldsymbol{Q}\left(\zeta_{2 m} \sqrt{b)}=\boldsymbol{Q}\left(\zeta_{m}, \zeta_{2 m} \sqrt{b)}=\right.\right.$ $\boldsymbol{Q}\left(\zeta_{m}, \zeta_{2 m}\right)=\boldsymbol{Q}\left(\zeta_{2 m}\right)$. Thus $\alpha=\zeta_{2 m} \sqrt{b}$ generates the splitting field of $x^{m}+b^{m / 2}$ so that the latter is p.n. with $s=2$.

On the other hand suppose that $\sqrt{b} \notin \boldsymbol{Q}\left(\zeta_{2 m}\right)$. Then $\boldsymbol{Q}\left(\zeta_{2 m} \sqrt{b}\right)=$ $\boldsymbol{Q}\left(\zeta_{m}, \zeta_{2 m} \sqrt{b}\right)$ is at most a quadratic extension of $\boldsymbol{Q}\left(\zeta_{m}\right)$. If, in fact, $\boldsymbol{Q}\left(\zeta_{2 m} \sqrt{b}\right)=\boldsymbol{Q}\left(\zeta_{m}\right) \varsubsetneqq \boldsymbol{Q}\left(\zeta_{2 m}\right)$, then $\sqrt{b} \in \boldsymbol{Q}\left(\zeta_{2 m}\right)$. Thus $\boldsymbol{Q}\left(\zeta_{2 m} \sqrt{b}\right)$ is an extension of degree 2 of $\boldsymbol{Q}\left(\zeta_{m}\right)$. As a result, $x^{m}+b^{m / 2}$ is p.n. with $s=2$ with splitting field generated by $\zeta_{2 m} \sqrt{b}$.

(6) In this case, $\alpha=\zeta_{m}^{3} \sqrt[4]{b}$ with $\boldsymbol{Q}\left(\zeta_{m}\right)=\boldsymbol{Q}\left(\alpha^{2}\right)=\boldsymbol{Q}\left(\zeta_{m / 2}^{j} \sqrt{b}\right)$. Thus $\sqrt{b} \in \boldsymbol{Q}\left(\zeta_{m}\right)$. Furthermore, $\sqrt{b} \notin \boldsymbol{Q}\left(\zeta_{m / 2}\right)$ and $4 \| m$ using a slight variation on the argument of Lemma 4.3 (B).

Conversely, suppose $4 \| m, \sqrt{b} \in \boldsymbol{Q}\left(\zeta_{m}\right)$ and $\sqrt{b} \notin \boldsymbol{Q}\left(\zeta_{m / 2}\right)$. Thus by 
Lemma 4.3 (B), $\boldsymbol{Q}\left(\zeta_{m / 2} \sqrt{b)}=\boldsymbol{Q}\left(\zeta_{m}\right)\right.$. Thus $\alpha=\zeta_{m} \sqrt[4]{b}$ generates the splitting field of $x^{m}-b^{m / 4}$ forcing the latter to be p.n. with $s=2$.

(7) If $x^{m}+b^{m / 4}$ is p.n. with $s=2$, then $\alpha=\zeta_{m}^{j} \zeta_{2 m} \sqrt[4]{b}$ and $\boldsymbol{Q}\left(\zeta_{m}\right)=\boldsymbol{Q}\left(\alpha^{2}\right)=\boldsymbol{Q}\left(\zeta_{m / 2}^{j} \zeta_{m} \sqrt{b)}\right.$. Thus $\sqrt{b} \in \boldsymbol{Q}\left(\zeta_{m}\right)$. We claim also that $\sqrt{b} \in \boldsymbol{Q}\left(\zeta_{m / 2}\right)$. For, if not, then by Lemma 4.3 (A), $\zeta_{m} \sqrt{b} \in \boldsymbol{Q}\left(\zeta_{m / 2}\right)$. It follows from this that

$$
\begin{aligned}
\boldsymbol{Q}\left(\zeta_{m}\right) & =\boldsymbol{Q}\left(\zeta_{m / 2}^{j} \zeta_{m} \sqrt{b}\right) \\
& =\boldsymbol{Q}\left(\zeta_{m / 2}, \zeta_{m} \sqrt{b}\right) \\
& =\boldsymbol{Q}\left(\zeta_{m / 2}\right)
\end{aligned}
$$

contradicting the fact that $4 \mid \mathrm{m}$.

Conversely, suppose that $\sqrt{b} \in \boldsymbol{Q}\left(\zeta_{m / 2}\right)$. Then $\zeta_{2 m} \sqrt[4]{b}$ is a root of $x^{2}-\zeta_{m} \sqrt{b}$ over $\boldsymbol{Q}\left(\zeta_{m}\right)$ and, therefore, $\boldsymbol{Q}\left(\zeta_{m}, \zeta_{2 m} \sqrt[4]{b}\right)$ is an extension of degree 2 over $\boldsymbol{Q}\left(\zeta_{m}\right)$. Furthermore, $\boldsymbol{Q}\left(\zeta_{m} \sqrt{b}\right)=\boldsymbol{Q}\left(\zeta_{m}\right)$ or $\boldsymbol{Q}\left(\zeta_{m / 2}\right)$. The latter cannot happen by Lemma $4.3(\mathrm{~A})$. Thus $\zeta_{2 m} \sqrt[4]{b}$ generates the splitting field of $x^{m}+b^{m / 4}$ with $s=2$.

(8) If $\alpha=\zeta_{m}^{j} \sqrt[6]{b}$ generates the splitting field of $x^{m}-b^{m / 6}$ with $\boldsymbol{Q}\left(\alpha^{3}\right)=\boldsymbol{Q}\left(\zeta_{m}\right)$, then $\sqrt{b} \in \boldsymbol{Q}\left(\zeta_{m}\right)$. Moreover, $\sqrt{b} \notin \boldsymbol{Q}\left(\zeta_{m / 3}\right)$ since otherwise $\boldsymbol{Q}\left(\alpha^{3}\right)=\boldsymbol{Q}\left(\zeta_{m / 3}^{j} \sqrt{b}\right) \leqq \boldsymbol{Q}\left(\zeta_{m / 3}\right)$. It follows from this that $3 \| m$. Furthermore, if $4 \mid m$ then $\left[\boldsymbol{Q}\left(\zeta_{m}\right): \boldsymbol{Q}\left(\zeta_{m / 6}\right)\right]=4$. But $\left[\boldsymbol{Q}\left(\alpha^{3}\right): \boldsymbol{Q}\left(\zeta_{m / 6}\right)\right]$ is at most 2. Thus also $2 \| m$.

Conversely, suppose $2\|m, 3\| m, \sqrt{b} \in \boldsymbol{Q}\left(\zeta_{m}\right)$ and $\sqrt{b} \notin \boldsymbol{Q}\left(\zeta_{m / 3}\right)$. Then Lemma 4.2 (B) applies and $\boldsymbol{Q}\left(\zeta_{m / 3} \sqrt{b}\right)=\boldsymbol{Q}\left(\zeta_{m}\right)$. Finally, $\boldsymbol{Q}\left(\zeta_{m} \sqrt[6]{b}\right)$ is of degree 3 over $\boldsymbol{Q}\left(\zeta_{m}\right)$. Thus $\zeta_{m} \sqrt[6]{b}$ generates the splitting field of $x^{m}-b^{m / 6}$ with $s=3$.

(9) If $\alpha=\zeta_{m}^{j} \zeta_{2 m} \sqrt[6]{b}$ generates the splitting field of $x^{m}+b^{m / 6}$ with $\boldsymbol{Q}\left(\alpha^{3}\right)=\boldsymbol{Q}\left(\zeta_{m}\right)$, then clearly $\sqrt{b} \notin \boldsymbol{Q}\left(\zeta_{m}\right)$ and $\sqrt{b} \in \boldsymbol{Q}\left(\zeta_{2 m}\right)$. Furthrmore, $\sqrt{b} \notin \boldsymbol{Q}\left(\zeta_{2 m / 3}\right)$ for, if otherwise, then

$$
\boldsymbol{Q}\left(\zeta_{m}\right)=\boldsymbol{Q}\left(\alpha^{3}\right)=\boldsymbol{Q}\left(\zeta_{m / 3}^{j} \zeta_{2 m / 3} \sqrt{b}\right) \leqq \boldsymbol{Q}\left(\zeta_{2 m / 3}\right)
$$

- clearly a contradiction.

Conversely, suppose $\sqrt{b} \in \boldsymbol{Q}\left(\zeta_{2 m}\right), \sqrt{b} \notin \boldsymbol{Q}\left(\zeta_{m}\right)$ and $\sqrt{b} \notin \boldsymbol{Q}\left(\zeta_{2 m / 3}\right)$. Then Lemma $4.2(\mathrm{~A})$ applies and

$$
\boldsymbol{Q}\left(\zeta_{2 m / 3} \sqrt{b}\right)=\boldsymbol{Q}\left(\zeta_{m}\right)
$$

Thus $x^{m}+b^{m / 6}$ is p.n. with $\alpha=\zeta_{2 m} \sqrt[6]{b}$. Since $\boldsymbol{Q}\left(\zeta_{2 m} \sqrt[6]{b}\right)$ is of degree 3 over $\boldsymbol{Q}\left(\zeta_{m}\right), s=3$.

This completes the proof of the theorem. 
We conclude this section with an example.

Lest one suspect that, if $x^{m}-b$ is partially normal, its splitting field can always be generated by $\alpha=\zeta_{m} \sqrt[m]{b}$, consider the polynomial $x^{12}-3^{6}$. According to Theorem 3.5 (3), this polynomial is partially normal with $\mathrm{s}=1, q=2$ and generating $\operatorname{root} \zeta_{12}^{j} \sqrt{3}$ for some positive integer $j$. Thus $\boldsymbol{Q}\left(\zeta_{12}^{j} \sqrt{3}\right)=\boldsymbol{Q}\left(\zeta_{12}\right)$. If $(j, 12)=2$, then by Lemma $4.3(\mathrm{~B}), \boldsymbol{Q}\left(\zeta_{12}^{j} \sqrt{3}\right)=\boldsymbol{Q}\left(\zeta_{12}\right)$ follows. However, if $(j, 12)=1, \zeta_{12}^{j} \sqrt{3}=$ $\left( \pm \zeta_{4}\right)\left( \pm \zeta_{3}\right) \sqrt{3}=( \pm 1 / 2) i(-1+\sqrt{-3}) \sqrt{3}=( \pm 1 / 2)(\sqrt{-3}+3)$. Thus $\boldsymbol{Q}\left(\zeta_{12}^{j} \sqrt{3}\right)=\boldsymbol{Q}\left(\zeta_{3}\right)$. Consequently, $\zeta_{12} \sqrt{3}$ is not a generating root for $x^{12}-3^{6}$ but $\zeta_{6} \sqrt{3}$ is!

6. Galois groups of partially normal binomials. In this section we will determine the Galois groups of the binomials listed in Theorem 3.5. We will assume the known facts about the Galois group of $\boldsymbol{Q}\left(\zeta_{n}\right)$ (see [4], Chapter 8): that $G\left(\boldsymbol{Q}\left(\zeta_{p^{n}}\right)\right)$ is cyclic of order $(p-1) p^{n-1}$ for $p$ an odd prime and that $G\left(\boldsymbol{Q}\left(\zeta_{m_{n}}\right)\right)=G\left(\boldsymbol{Q}\left(\zeta_{m}\right)\right) \times G\left(\boldsymbol{Q}\left(\zeta_{n}\right)\right)$ wherever $(n, m)=1$. We will also assume the Galois theoretic fact ([4] p. 196) that if $A$ and $B$ are two Galois extensions of $C$ with groups $G$ and $H$ respectively, then the group (over $C$ ) of the compositum $A B$ is $G \times H$ iff $A \cap B=C$.

The following theorem determines the groups of the binomials of 3.5 except cases (6) and (7) (which will be treated separately).

THEOREM 6.1. Let $G\left(x^{m}-a\right)$ denote the group of the partially normal binomial $x^{m}-a$ over $\boldsymbol{Q}$. Then the following are the Galois groups numbered according to the scheme of 3.5:

(1) $G\left(x^{m}-b^{m}\right) \cong G\left(x^{m}-1\right) ; G\left(x^{m}+b^{m}\right) \cong G\left(x^{2 m}-1\right)$.

(2) $G\left(x^{m}-b^{m / 2}\right) \cong G\left(x^{2 m}-1\right)$.

(3) $G\left(x^{m}-b^{m / 2}\right) \cong G\left(x^{m}-1\right)$.

(4) $G\left(x^{m}+b^{m / 2}\right) \cong G\left(x^{m}-1\right)$.

(5) $G\left(x^{m}+b^{m / 2}\right) \cong G\left(x^{2 m}-1\right)$.

(8) $G\left(x^{m}-b^{m / 6}\right) \cong S_{3} \times G\left(x^{m}-1\right)$.

(9) $G\left(x^{m}+b^{m / 6}\right) \cong S_{3} \times G\left(x^{m / 3}-1\right)$.

Here $S_{3}$ denotes the symmetric group on 3 letters.

Proof. (1), (3) and (4) are clear.

(2) In this case, $\boldsymbol{Q}(\alpha)=\boldsymbol{Q}\left(\zeta_{m} \sqrt{b}\right)=\boldsymbol{Q}\left(\zeta_{m}, \sqrt{b}\right)$. Since $\sqrt{b} \notin$ $\boldsymbol{Q}\left(\zeta_{m}\right)$, the group of $\boldsymbol{Q}(\alpha)$ is isomorphic to $\boldsymbol{Z}_{2} \times G\left(x^{m}-1\right)$ which, since $2 \| m$, is isomorphic to $G\left(x^{2 m}-1\right)$.

(5) For this case, either $\sqrt{\bar{b}} \in \boldsymbol{Q}\left(\zeta_{m}\right)$ or $\sqrt{b} \notin \boldsymbol{Q}\left(\zeta_{2 m}\right)$. In the first instance, $\boldsymbol{Q}(\alpha)=\boldsymbol{Q}\left(\zeta_{2 m} \sqrt{b}\right)=\boldsymbol{Q}\left(\zeta_{m}, \zeta_{2 m} \sqrt{b}\right)=\boldsymbol{Q}\left(\zeta_{m}, \zeta_{2 m}\right)=\boldsymbol{Q}\left(\zeta_{2 m}\right)$. Thus the group is $G\left(x^{2 m}-1\right)$. In the second, let $m=2^{k} q, q$ odd. Then 
$\boldsymbol{Q}(\alpha)=\boldsymbol{Q}\left(\zeta_{2 m} \sqrt{b}\right)=\boldsymbol{Q}\left(\zeta_{q}, \zeta_{2^{k}}, \zeta_{2^{k+1}} \sqrt{b}\right)$. Thus the group of $\boldsymbol{Q}(\alpha)$ is isomorphic to $G\left(x^{q}-1\right) \times G\left(\boldsymbol{Q}\left(\zeta_{2^{k}}, \zeta_{2^{k+1}} \sqrt{b}\right)\right)$. The second factor is $G\left(x^{2^{k}}+b^{2^{k-1}}\right)$ which for $k \geqq 3$ is isomorphic, by ([4], Theorem 9), to $\boldsymbol{Z}_{2} \times \boldsymbol{Z}_{2^{k-1}}$. For $k=1,2$ it is easy to check that the same result holds. Hence $G\left(x^{m}+b^{m / 2}\right) \cong G\left(x^{2 m}-1\right)$ in the second instance also.

(8) Since in this case $2\|m, 3\| m$, and $\sqrt{b} \in \boldsymbol{Q}\left(\zeta_{m}\right)$, we have $\boldsymbol{Q}(\alpha)=\boldsymbol{Q}\left(\zeta_{m}, \sqrt[6]{b}\right)=\boldsymbol{Q}\left(\zeta_{m},(\sqrt[3]{b})^{-1} \sqrt{b}\right)=\boldsymbol{Q}\left(\zeta_{m}, \sqrt[3]{b}\right)=\boldsymbol{Q}\left(\zeta_{m / 3}, \zeta_{3}, \sqrt[3]{b}\right)$. Thus since $\boldsymbol{Q}\left(\zeta_{m / 2}\right) \cap \boldsymbol{Q}\left(\zeta_{3}, \sqrt[3]{b}\right)=\boldsymbol{Q}$ and $\boldsymbol{Q}\left(\zeta_{3}, \sqrt[3]{b}\right)$ has group $S_{3}$, we conclude that $G\left(x^{m}-b^{m / 6}\right) \cong S_{3} \times G\left(x^{m / 3}-1\right)$.

(9) If $x^{m}+b^{m / 6}$ is p.n., then $\alpha=\zeta_{2 m} \sqrt[6]{b}$ and therefore $\zeta_{m} \sqrt[3]{b} \epsilon$ $\boldsymbol{Q}(\alpha)$. Since also $\zeta_{m} \in \boldsymbol{Q}(\alpha)$, we must have that $\sqrt[3]{b} \in \boldsymbol{Q}(\alpha)$. Consequently, $\boldsymbol{Q}\left(\zeta_{3}, \sqrt[3]{b}\right) \subset \boldsymbol{Q}(\alpha)$. Furthermore, $\boldsymbol{Q}\left(\zeta_{3}, \sqrt[3]{b}\right) \cap \boldsymbol{Q}\left(\zeta_{m / 3}\right)=\boldsymbol{Q}$ and $\left[\boldsymbol{Q}\left(\zeta_{3}, \sqrt[3]{b}\right): \boldsymbol{Q}\right] \cdot\left[\boldsymbol{Q}\left(\zeta_{m / 3}\right): \boldsymbol{Q}\right]=3 \cdot \phi(m)$ since $3 \| m$. Hence $\boldsymbol{Q}(\boldsymbol{\alpha})=\boldsymbol{Q}\left(\zeta_{m / 3}\right.$, $\left.\zeta_{3}, \sqrt[3]{b}\right)$ with Galois group $S_{3} \times G\left(x^{m / 3}-1\right)$.

The remainder of this section will be devoted to calculating the groups of (6) and (7) of 3.5. To do this we will use the following lemma and its corollaries.

LEMMA 6.2. Let $a$ and $b$ be relatively prime square free integers. Suppose that $A$ and $B$ are cyclic fields of degrees $2^{i}$ and $2^{j}$ over $A \cap B=\boldsymbol{Q}$ such that $\sqrt{a} \in A, \sqrt{b} \in B$. If $i \leqq j$, then there is $a$ cyclic field $C$ of degree $2^{i}$ over $\boldsymbol{Q}$ with $\sqrt{a b} \in C, C B=A B$ and $C \cap B=\boldsymbol{Q}$.

Proof. Let $G_{A}, G_{B}$ be the groups of $A, B$ respectively. Then the group of $A B$ is $G_{A} \times G_{B}$. If $\sigma, \tau$ generates $G_{A}, G_{B}$ respectively, then $\sigma(\sqrt{a})=-\sqrt{a}$ and $\tau(\sqrt{b})=-\sqrt{b}$. The subgroup $H$ of $G_{A} \times G_{B}$ generated by $(\sigma, \tau)$ is cyclic of order $2^{j}$ and fixes $\sqrt{a b}$. Let $C$ be the fixed field of $H$. Then $\sqrt{a b} \in C, G_{A} \times H=G_{A} \times G_{B}, C$ has group $G_{A}$ and the fixed field of $G_{A}$ is $B$ with group $H \cong G_{B}$. The lemma follows.

COROLlaRY 6.3. Let $a_{1}, \cdots, a_{n}$ be pairwise relatively prime square free integers, $K_{i}$ a cyclic field of degree $2^{j_{i}}$ with $\sqrt{a_{i}} \epsilon$ $K_{i}(i=1, \cdots, n)$ arranged so that $j_{1} \leqq j_{2} \leqq \cdots \leqq j_{n}$. Assume that $K_{i} \cap K_{j}=\boldsymbol{Q}$ whenever $i \neq j$. Then there exists a cyclic field $K_{0}$ of degree $2^{j_{1}}$ with $\sqrt{a_{1} a_{2} \cdots a_{n}} \in K_{0}$ and a field $K$ such that $K_{0} \cap K=\boldsymbol{Q}$ and $K_{0} K=K_{1} K_{2} \cdots K_{n}$.

Proof. Use the lemma and induction on $n$ : successively construct pairs of fields $J_{i}, L_{i}$ such that $J_{i}$ is cyclic of degree $2^{j_{1}}$ containing $\sqrt{a_{1} a_{2} \cdots a_{i}}, J_{i} \cap L_{i}=Q$ and $J_{i} L_{i}=K_{1} K_{2} \cdots K_{i}$. Then $K_{0}=J_{n}, K=$ $L_{n}$ satisfy the conclusion of the corollary. 
COROLlaRY 6.4. Let $m$ and $b$ be positive integers such that $m$ is odd and $b$ is square free. Let $b=p_{1} p_{2} \cdots p_{n}$ be the prime factorization of $b$ and, for $i=1, \cdots, n$, let $j_{i}$ be the positive integer such that $2^{j_{i}} \| p_{i}-1$. By relabelling, if necessary, assume that $j_{1} \leqq$ $j_{2} \leqq \cdots \leqq j_{n}$. Then, if $\sqrt{b} \in \boldsymbol{Q}\left(\zeta_{m}\right)$, there exist subfields $F_{0}, F$ of $\boldsymbol{Q}\left(\zeta_{m}\right)$ such that $F_{0}$ is cyclic of degree $2^{j_{1}}, \sqrt{b} \in F_{0}, F_{0} \cap F=\boldsymbol{Q}$ and $F_{0} F=\boldsymbol{Q}\left(\zeta_{m}\right)$.

Proof. By Lemma 4.1, $b \mid m$. Let $d$ be the largest divisor of $m$ relatively prime to $b$. Then $G\left(x^{m}-1\right)=G\left(x^{d}-1\right) \times G\left(x^{m / d}-1\right)$. Let $G=G\left(x^{m / d}-1\right), G_{2}$ its Sylow 2-subgroup, and $G_{1}$, the direct product of its Sylow $p$-subgroups, $p$ an odd prime. Then $G=G_{2} \times G_{1}$. Let $J_{2}, J_{1}$, be the fixed fields of $G_{1}, G_{2}$ respectively. It is easy to see that $\sqrt{b} \in J_{2}$ and that $J_{2}$ is the compositum of pairwise linearly disjoint cyclic fields $K_{i}$ with $\sqrt{ \pm p_{i}} \in K_{i}$ and $\left[K_{i}: Q\right]=2^{j i}(i=1, \cdots, n)$. The hypotheses of Corollary 6.3 are thus satisfied so that we can find $K_{0}$, $K \subset J_{2}$ with $K_{0}$ cyclic of degree $2^{j_{1}}, K_{0} \cap K=\boldsymbol{Q}$ and $K_{0} K=J_{2}$.

The corollary follows with $F_{0}=K_{0}$ and $F=K J_{1} \boldsymbol{Q}\left(\zeta_{d}\right)$.

We can now compute the group of case (6) of Theorem 3.5.

THEOREM 6.5. Let $x^{m}-b^{m / 4}$ be a partially normal binomial of type (6). Let $D_{4}$ denote the dihedral group on four letters. Then there exists a direct cyclic factor $H$ of $G\left(x^{m / 4}-1\right)$ of order 2 such that $G\left(x^{m}-b^{m / 4}\right) \cong D_{4} \times\left(G\left(x^{m / 4}-1\right) / H\right)$.

Proof. Let $m=4 q$ with $q$ odd. Then

$$
\boldsymbol{Q}(\alpha)=\boldsymbol{Q}\left(\zeta_{4 q} \sqrt[4]{b}\right)=\boldsymbol{Q}\left(\zeta_{q}, \zeta_{4}, \sqrt[4]{b)}\right.
$$

By Theorem 3.5, $\sqrt{b} \in \boldsymbol{Q}\left(\zeta_{4 q}\right)$ but $\sqrt{b} \notin \boldsymbol{Q}\left(\zeta_{q}\right)$. Thus $\sqrt{-b} \in\left(\zeta_{q}\right)$ and $\boldsymbol{Q}\left(\zeta_{q}\right) \cap \boldsymbol{Q}\left(\zeta_{4}, \sqrt[4]{b}\right)=\boldsymbol{Q}(\sqrt{-b})$. By Lemma 4.1 the integer $j_{1}$ of Corollary 6.4 must be 1 . Hence, by the same corollary, there is a field $F \subset \boldsymbol{Q}\left(\zeta_{q}\right)$ with $\boldsymbol{Q}(\sqrt{-b}) \cdot \boldsymbol{F}=\boldsymbol{Q}\left(\zeta_{q}\right)$ and $\boldsymbol{Q}(\sqrt{-b}) \cap F=\boldsymbol{Q}$. Thus $\boldsymbol{Q}(\alpha)=F \cdot \boldsymbol{Q}\left(\zeta_{4}, \sqrt[4]{b}\right)$ and $F \cap \boldsymbol{Q}\left(\zeta_{4}, \sqrt[4]{b}\right)=\boldsymbol{Q}$. The theorem follows.

We now turn to case (7) of Theorem 3.5. Let $p(x)=x^{2^{k_{m}}}+b^{2^{k-2} m}$ be a fixed partially normal binomial with $m$ odd, $b$ a square free integer and $k \geqq 2$. Thus, by $3.5, \sqrt{b} \in \boldsymbol{Q}\left(\zeta_{2^{k-1} m}\right)$ and $\alpha=\zeta_{2^{k+1} m} \sqrt[4]{b}$. It is clear that $\boldsymbol{Q}(\alpha)=\boldsymbol{Q}\left(\zeta_{m}, \zeta_{2^{k+1}} \sqrt[4]{b)}\right.$.

To compute $G(p(x))$, let $b=2^{\varepsilon_{0}} b_{1}$ where $\varepsilon_{0}=0$ or 1 and $b_{1}$ is odd. Let $\delta=0$ or 1 so that $i^{\delta} \sqrt{b_{1}} \in \boldsymbol{Q}\left(\zeta_{m}\right)$ (by 4.1). Then by Corollary 6.4 
there are fields $F_{0}, F \subset \boldsymbol{Q}\left(\zeta_{m}\right)$ so that $F_{0} \boldsymbol{F}=\boldsymbol{Q}\left(\zeta_{m}\right), G\left(\boldsymbol{Q}\left(\zeta_{m}\right)\right)=G\left(F_{0}\right) \times$ $G(F), F_{0}$ is cyclic of degree $2^{j_{1}}$ and $i^{\delta} \sqrt{b_{1}} \in F_{0}$. Thus it is easy to see that $G(p(x))=G\left(F_{0}\left(\zeta_{2^{k+1}} \sqrt[4]{b}\right)\right) \times G(F)$. Since $G(F)$ can be computed from 6.4, it is sufficient to determine $G\left(F_{0}\left(\zeta_{2^{k+1}} \sqrt[4]{b}\right)\right.$ ).

Let $\beta=\zeta_{2^{k+1}} \sqrt[4]{b}$. We know that $F_{0}(\beta)$ is an extension of degree 2 over $F_{0}\left(\zeta_{2^{k}}\right)$ so that there is an automorphism $\sigma$ of $F_{0}(\beta)$ such that $\sigma(\beta)=-\beta$ with fixed field $F_{0}\left(\zeta_{2^{k}}\right)$. On the other hand, the group of $F_{0}\left(\zeta_{2_{k}}\right)$ is the direct product of cyclic groups of orders $2^{j_{1}}, 2,2^{k-2}$ with respective generators $\rho, \sigma_{1}, \sigma_{2}$. Denoting by the same letters extensions of these generators to $F_{0}(\beta)$, we have that the group $G^{\prime}$ of $F_{0}(\beta)$ is generated by $\sigma, \rho, \sigma_{1}, \sigma_{2}$. On $F_{0}\left(\zeta_{2^{k}}\right)$ these generators are defined by

$$
\begin{aligned}
& \rho\left(\zeta_{2^{k}}\right)=\zeta_{2^{k}}, \rho(\sqrt{b})=(-1)^{\varepsilon_{1}} \sqrt{b} ; \\
& \sigma_{2} \mid F_{0}=1, \sigma_{2}\left(\zeta_{2^{k}}\right)=\zeta_{2^{k}}^{-1}, \sigma_{2}(\sqrt{b})=\sqrt{b} ; \\
& \sigma_{1} \mid F_{0}=1, \sigma\left(\zeta_{2^{k}}\right)=\zeta_{2}^{5}, \sigma_{1}(\sqrt{b})=(-1)^{\varepsilon_{0} 0} \sqrt{b}
\end{aligned}
$$

where $\varepsilon_{0}$ is as defined above and $\varepsilon_{1}=1$ if $b$ is odd or $\varepsilon_{0}=1$ and $b / 2>1$ and 0 otherwise. To see how $\rho, \sigma_{1}, \sigma_{2}$ may be extended to $F_{0}(\beta)$, it is sufficient to determine how they act on $\beta$ :

$$
\begin{aligned}
& \rho(\beta)=i^{\varepsilon_{1}} \beta, \\
& \sigma_{1}(\beta)=\zeta_{2^{k}} i^{\varepsilon_{0}} \beta, \\
& \sigma_{2}(\beta)=\zeta_{2^{k}}^{-1} \beta .
\end{aligned}
$$

From these formulas, it is a straightforward matter to determine relations amongst the generators $\sigma, \rho, \sigma_{1}$, and $\sigma_{2}$. For one, $\sigma_{1}^{2 k-2}=\sigma$. We summarize the rest in the following.

THEOREM 6.6. The group $G\left(x^{2^{k} m}+b^{2 k-1} m\right)$ is isomorphic to the direct product of an abelian group $G(F)$ with a nonabelian group $G^{\prime}$ of order $2^{j_{1}+k}$ generated by $\rho, \sigma_{1}, \sigma_{2}$ and satisfying the following relations:

$$
\begin{aligned}
& \sigma_{2}^{2^{k-1}}=\sigma_{2}^{2}=\rho^{a}=1 \quad\left(\text { where } a=\max \left(2^{j_{1}}, 4^{\varepsilon_{1}}\right)\right), \\
& \sigma_{1} \sigma_{2}=\sigma_{1}^{\varepsilon_{0} 2^{k-2}+1} \sigma_{2}, \\
& \sigma_{2} \rho=\rho \sigma_{2} \sigma_{1}^{\varepsilon_{1} 2^{k-2}}, \\
& \sigma_{1} \rho=\rho \sigma_{1} .
\end{aligned}
$$

In particular,

(a) if $\varepsilon_{1}=0$, then $j_{1}=0$ and $G(F)=G\left(x^{m}-1\right)$; furthermore $G^{\prime}$ $i s$ isomorphic to a group generated by $\sigma_{1}, \sigma_{2}$, satisfying relations $\sigma_{1}^{2^{k-1}}=\sigma_{2}^{2}=1, \sigma_{2} \sigma_{1}=\sigma_{1}^{2^{k-2+1}} \sigma_{2} ;$ 
(b) if $\varepsilon_{0}=\varepsilon_{1}=1$ and $j_{1} \geqq k-1$, then $G(p(x))$ is isomorphic to $G\left(x^{m}-1\right) \times\left\langle\sigma_{1}, \sigma_{2}\right\rangle$.

Proof. The relations $\left({ }^{*}\right)$ are easily determined from the discussion preceeding the theorem. Part (a) follows from $\left(^{*}\right)$ and the fact that $b=2$ in this case. Finally, (b) follows from (*) by setting $\tau=\rho \sigma_{1}$. Then $\sigma_{2} \tau=\tau \sigma_{2}$ and $\tau$ is an element of order $2^{j_{1}}$. By Corollary 6.4, $\langle\tau, G(F)\rangle \cong G\left(x^{m}-1\right)$. Furthermore, $\langle\tau\rangle \cap\left\langle\sigma_{1}, \sigma_{2}\right\rangle=\{1\}$ and thus $G(p(x)) \cong\left\langle\sigma_{1}, \sigma_{2}\right\rangle \times G\left(x^{m}-1\right)$.

\section{REFERENCES}

1. Giulio Darbi, Sulla riducibilità delle equazione algebriche, Annali di Mat. pur. e appl., Ser. 4, 4 (1926), 185-208.

2. Lisl. Gaal, Classical Galois Theory, Chelsea, New York, 1973.

3. D. Gay, On normal radical extensions of real fields, to appear, Acta Arithmetica, 35 (1978).

4. Serge Lang, Algebra, Addison-Wesley, Reading, Mass., 1965.

5. Henry B. Mann and William Yslas Vélez, On normal radical extensions of the rationals, J. of Lin. and Multilin. Alg., 3 (1975), 73-80.

6. M. J. Norris and W. Y. Vélez, Structure theorems for radical extensions of fields, Sandia Laboratories Appl. Math. Report, 1976.

7. A. Schinzel, Abelian binomials, power residues and exponential congruences, Acta Arithmetica, 32 (1977), 245-274.

8. N. G. Tschebotarev and H. Schwerdtfeger, Grundzüge der Galoischen Theorie, Groningen-Djakarta, 1950.

Received December 9, 1976. The first author was supported in part by the Fonds National Suisse and Battelle Institute Grant No: 333-205. The third author was supported in part by a fellowship from the Ford Foundation and by the U.S. Energy and Research Development Administration (ERDA).

UNIVERSITY OF ARIZONA,

TUSCON, AZ 85721

AND

New College

SARASOTA, FL 33580

AND

SANDIA LABORATORIES

Albuquerque, NM 87115 



\title{
PACIFIC JOURNAL OF MATHEMATICS
}

\section{EDITORS}

\author{
RICHARD ARENS (Managing Editor) \\ University of California \\ Los Angeles, CA 90024 \\ Charles W. Curtis \\ University of Oregon \\ Eugene, OR 97403 \\ C. C. MOORE \\ University of California \\ Berkeley, CA 94720
}

\section{J. DUGUNDJI}

Department of Mathematics

University of Southern California

Los Angeles, CA 90007

R. FINN and J. MILGRAM

Stanford University

Stanford, CA 94305

\section{ASSOCIATE EDITORS}
E. F. BECKENBACH
B. H. NeumanN
F. WOLF
K. YoSHIDA

\section{SUPPORTING INSTITUTIONS}

UNIVERSITY OF BRITISH COLUMBIA

CALIFORNIA INSTITUTE OF TECHNOLOGY

UNIVERSITY OF CALIFORNIA

MONTANA STATE UNIVERSITY

UNIVERSITY OF NEVADA, RENO

NEW MEXICO STATE UNIVERSITY

OREGON STATE UNIVERSITY

UNIVERSITY OF OREGON

OSAKA UNIVERSITY

\author{
UNIVERSITY OF SOUTHERN CALIFORNIA \\ STANFORD UNIVERSITY \\ UNIVERSITY OF HAWAII \\ UNIVERSITY OF TOKYO \\ UNIVERSITY OF UTAH \\ WASHINGTON STATE UNIVERSITY \\ UNIVERSITY OF WASHINGTON \\ $\stackrel{*}{*} \stackrel{*}{*} \stackrel{*}{*}$ AMERICAN MATHEMATICAL SOCIETY
}

The Supporting Institutions listed above contribute to the cost of publication of this Journal, but they are not owners or publishers and have no responsibility for its content or policies.

Mathematical papers intended for publication in the Pacific Jaurnal of Mathematics should be in typed form or offset-reproduced, (not dittoed), double spaced with large margins. Please do not use built up fractions in the text of your manuscript. You may however, use them in the displayed equations. Underline Greek letters in red, German in green, and script in blue. The first paragraph or two must be capable of being used separately as a synopsis of the entire paper. Items of the bibliography should not be cited there unless absolutely necessary, in which case they must be identified by author and Journal, rather than by item number. Manuscripts, in triplicate, may be sent to any one of the editors. Please classify according to the scheme of Math. Reviews, Index to Vol. 39. All other communications should be addressed to the managing editor, or Elaine Barth, University of California, Los Angeles, California, 90024.

The Pacific Journal of Mathematics expects the author's institution to pay page charges, and reserves the right to delay publication for nonpayment of charges in case of financial emergency.

100 reprints are provided free for each article, only if page charges have been substantially paid. Additional copies may be obtained at cost in multiples of 50 .

The Pacific Journal of Mathematics is issued monthly as of January 1966. Regular subscription rate: $\$ 7200$ a year (6 Vols., 12 issues). Special rate: $\$ 36.00$ a year to individual members of supporting institutions.

Subscriptions, orders for back numbers, and changes of address should be sent to Pacific Journal of Mathematics, 103 Highland Boulevard, Berkeley, California, 94708.

PUBLISHED BY PACIFIC JOURNAL OF MATHEMATICS, A NON-PROFIT CORPORATION

Printed at Kokusai Bunken Insatsusha (International Academic Printing Co., Ltd.). 8-8, 3-chome, Takadanobaba, Shinjuku-ku, Tokyo 160, Japan.

Copyright (C) 1975 by Pacific Journal of Mathematics Manufactured and first issued in Japan 


\section{Pacific Journal of Mathematics}

Vol. 72, No. 2

February, 1977

George E. Andrews, Plane partitions. II. The equivalence of the

Bender-Knuth and MacMahon conjectures ................. 283

Lee Wilson Badger, An Ehrenfeucht game for the multivariable quantifiers

of Malitz and some applications ......................... 293

Wayne C. Bell, A decomposition of additive set functions ............ 305

Bruce Blackadar, Infinite tensor products of $C^{*}$-algebras ............. 313

Arne Brøndsted, The inner aperture of a convex set .............. 335

N. Burgoyne, Finite groups with Chevalley-type components........... 341

Richard Dowell Byrd, Justin Thomas Lloyd and Roberto A. Mena, On the retractability of some one-relator groups .....................

Paul Robert Chernoff, Schrödinger and Dirac operators with singular potentials and hyperbolic equations .................... 361

John J. F. Fournier, Sharpness in Young's inequality for convolution ....... 383

Stanley Phillip Franklin and Barbara V. Smith Thomas, On the metrizability

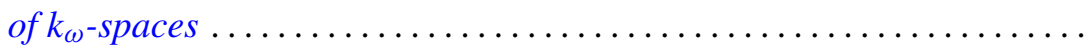

David Andrew Gay, Andrew McDaniel and William Yslas Vélez, Partially normal radical extensions of the rationals .................... 403

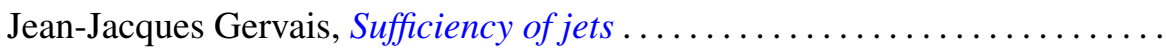

Kenneth R. Goodearl, Completions of regular rings. II . .

Sarah J. Gottlieb, Algebraic automorphisms of algebraic groups with stable maximal tori

Donald Gordon James, Invariant submodules of unimodular Hermitian forms.....

J. Kyle, $W_{\delta}(T)$ is convex.

Ernest A. Michael and Mary Ellen Rudin, A note on Eberlein compacts ...

Ernest A. Michael and Mary Ellen Rudin, Another note on Eberlein compacts ....

Thomas Bourque Muenzenberger and Raymond Earl Smithson, Fixed point theorems for acyclic and dendritic spaces.

Budh Singh Nashier and A. R. Rajwade, Determination of a unique solution of the quadratic partition for primes $p \equiv 1(\bmod 7)$.

Frederick J. Scott, New partial asymptotic stability results for nonlinear ordinary differential equations ....................

Frank Servedio, Affine open orbits, reductive isotropy groups, and dominant gradient morphisms; a theorem of Mikio Sato..........

D. Suryanarayana, On the distribution of some generalized square-full integers.................................. 